\title{
BMJ Open Acceptability of a perturbation-based balance training programme for falls prevention in older adults: a qualitative study
}

\author{
Marissa H G Gerards (D) , ${ }^{1,2}$ Judith Sieben, ${ }^{2,3}$ Rik Marcellis, ${ }^{1}$ Rob A de Bie, ${ }^{2}$ \\ Kenneth Meijer, ${ }^{4}$ Antoine F Lenssen (D) ${ }^{1}$
}

To cite: Gerards MHG, Sieben J, Marcellis R, et al. Acceptability of a perturbation-based balance training programme for falls prevention in older adults: a qualitative study. BMJ Open 2022;12:e056623. doi:10.1136/ bmjopen-2021-056623

- Prepublication history and additional supplemental material for this paper are available online. To view these files, please visit the journal online (http://dx.doi.org/10.1136/ bmjopen-2021-056623)

Received 20 September 2021 Accepted 28 January 2022

A Check for updates

(c) Author(s) (or their employer(s)) 2022. Re-use permitted under CC BY-NC. No commercial re-use. See rights and permissions. Published by BMJ.

For numbered affiliations see end of article.

Correspondence to Marissa H G Gerards; marissa.gerards@ maastrichtuniversity.n

\section{ABSTRACT}

Introduction Perturbation-based balance training (PBT) is reported to effectively reduce falls in older adults and may even be superior compared with various exercise programmes. Due to the nature of the intervention, requiring unpredictable balance perturbations, the question arises whether acceptability is an issue in PBT.

Objective To evaluate the acceptability of PBT in older adults with a recent history of falls.

Design, method, participants and setting This is a qualitative study in which semistructured interviews were conducted in 16 older adults (14 women and 2 men, mean age 73.6 \pm 6.0 years) who completed a three-session PBT protocol as part of another study in a university medical centre in the Netherlands. Typical case and purposive sampling strategies were applied. Interviews were based on the theoretical framework of acceptability (TFA) alongside context-specific factors and analysed using a template analysis approach.

Results The results indicate that this PBT protocol is perceived as acceptable by older adults with a recent history of falls and highlight key areas for potential future modifications. Enjoyment of the novel training and technology, being able to feel safe during training, and perceived impact of increased self-efficacy and balance confidence were identified as facilitating factors. Potential issues included initial apprehension or anxiety during training and perceived impact being predominantly psychological instead of physical. Complementary to the TFA one additional theme emerged which described challenges regarding the training setting, such as preference for group training in some participants and travel to the training location.

Conclusions The results suggest that PBT is perceived acceptable by older adults with a history of falls. Increasing the social aspect of training and sharing the experiences of peers may be considered to enhance acceptability to new participants who initially feel apprehensive or anxious about their ability to participate in future implementation of PBT.

Trial registration number The article is linked to a randomised clinical trial registered on https://www. trialregister.nl/trial/7680, NL7680; Results.
Strengths and limitations of this study

- This is the first study to use the theoretical framework of acceptability (TFA) to explore older adults' perceived acceptability of perturbation-based balance training (PBT).

- Using the TFA enabled a systematic approach to define and assess intervention acceptability.

- Triangulation was applied in data collection as well as data analysis to increase trustworthiness of the research findings.

- The PBT intervention was applied in a research setting, meaning that some specific factors, such as willingness to pay for participation in the intervention, were not evaluated.

- The results only reflect the perceived retrospective acceptability of the PBT and may not entirely reflect how participants' views changed over time.

\section{INTRODUCTION}

Falls in community-dwelling older adults can be effectively reduced through exercise interventions including balance training. ${ }^{1}$ However, the search for the optimal balance training programme for falls prevention is still ongoing. With conventional balance training, a relatively high number of training sessions are needed, discontinuation rates are high and retention of training effects is hard to accomplish. $^{2} 3$ Moreover, conventional balance training seems not sufficiently taskspecific to prevent falls due to slips or trips during walking, which cause up to $60 \%$ of falls in community-dwelling older adults. ${ }^{45}$ It is not likely that conventional balance training, mostly targeting volitional movements, will improve the change-in-support reactions (eg, taking a quick step) needed to prevent a slip or a trip due to the additional speed and stability requirements of these balance reactions. ${ }^{6}$ Therefore, an increasing interest has arisen in more task-specific balance training 
interventions, such as perturbation-based balance training (PBT).

PBT aims to improve rapid balance reactions after unexpected external perturbations. During PBT, participants are repeatedly exposed to destabilising perturbations while performing activities of daily living in a safe and controlled environment. A systematic review by Mansfield et $a l^{7}$ indicated that PBT may be more effective in reducing daily-life falls in older adults compared with various interventions ranging from no exercise to individualised physical therapy (risk ratio $0.71,95 \%$ CI 0.52 to $0.96 ; \mathrm{p}=0.02)$. With PBT, balance adaptation may occur faster, potentially achieving equal or better results with fewer training sessions compared with conventional balance training. ${ }^{8}$

However promising, even effective interventions are likely to fail if they are not acceptable to the target population. The more acceptable the intervention, the more likely that adherence will be high. ${ }^{9}$ In turn, higher adherence $(>80 \%)$ may result in larger effects. ${ }^{10}$ Due to the nature of the intervention, the question arises whether acceptability is an issue in PBT. In 2019, Okubo et $a l^{11}$ found in a pilot study of 10 healthy older adults that self-reported anxiety levels before a training session increased significantly with increasing unpredictability of PBT. Unpredictable perturbations are required in PBT to maximise learning of reactive balance control, yet these perturbations may cause anxiety and consequentially decrease acceptability.

So far limited evidence exists about the acceptability of PBT. Previous studies reported high training adherence rates and no significant differences in dropout rates between PBT and control groups receiving exercise or flexibility training. ${ }^{12-14}$ However, while quantitative data such as adherence rates may be indirect indicators of acceptability, the full-fledged concept of acceptability is a subjective evaluation made by individuals who experience an intervention. ${ }^{9}$ In 2017 , Sekhon et at ${ }^{9}$ proposed the theoretical framework of acceptability (TFA), in which acceptability is viewed as a multifaceted construct, consisting of seven components.

To our knowledge, no studies thus far have focused on the qualitative aspects of acceptability of PBT in older adults. The aim of this study is to explore the acceptability of PBT as perceived by older adults with a recent history of falls. Their views on the components constituting acceptability will be explored. The findings will enable optimisation of future implementation of PBT in clinical practice.

\section{METHODS}

\section{Study design}

A qualitative study consisting of semistructured interviews was conducted. All participants provided written informed consent. The study is reported in line with the Consolidated criteria for Reporting Qualitative research statement. $^{15}$

\section{Context, study participants and sampling}

Older adults who participated in PBT as part of a randomised controlled trial (RCT) were included. ${ }^{16}$ Community-dwelling older adults (age $\geq 65$ years) who visited the hospital's outpatient clinic due to a fall incident were informed about the study and approached by telephone 3-7 days later. Participants were included in the RCT if they were able to walk for $15 \mathrm{~min}$ without a walking aid. Exclusion criteria included any risk factors to them participating in PBT (eg, diagnosed osteoporosis, severe cardiopulmonary disease) or inability to communicate in Dutch. Participants were eligible for the qualitative interviews after they had completed the PBT. They were selected by the PBT trainers through a combination of typical case and purposive sampling to select those participants who were representative of the study population and were expected to provide the most detailed input.

The PBT protocol consisted of three $30 \mathrm{~min}$ sessions, during which participants were exposed to sudden balance perturbations while they stood and walked on a dual-belt treadmill embedded in a moveable platform (Computer Assisted Rehabilitation Environment (CAREN), Motek Medical). During the training, virtual environments were projected on a $180^{\circ}$ screen in front of the platform. Each session consisted of three standardised conditions, while progression of difficulty levels in each condition was individualised.

- Gait adaptability: participants walked on the treadmill while a virtual environment of a forest road, with various slopes and turns, was projected on to the screen. The platform moved correspondingly.

- Static reactive balance: participants stood on the platform while the platform and treadmill made sudden, variable and unpredictable movements to perturb balance.

- Dynamic reactive balance: this training condition was similar to the one above; only the perturbations were applied while the participant was walking on the treadmill.

A detailed description of the PBT protocol is published elsewhere. ${ }^{16}$ For an impression of the PBT setting, see figure 1 .

\section{Theoretical framework}

The interviews were based on the TFA as proposed by Sekhon et $a l .{ }^{9}$ In this framework, acceptability is defined as a multifaceted, seven-component construct, including affective attitude, burden, ethicality, intervention coherence, opportunity costs, perceived effectiveness and self-efficacy. Table 1 provides an overview of our operationalisation of each construct and an example of a related question from our interview guide (online supplemental file 1). Questions regarding context-specific barriers and facilitators (eg, training location, supervision during training) to participate in PBT were added to gain insight into their influence on PBT acceptability. 


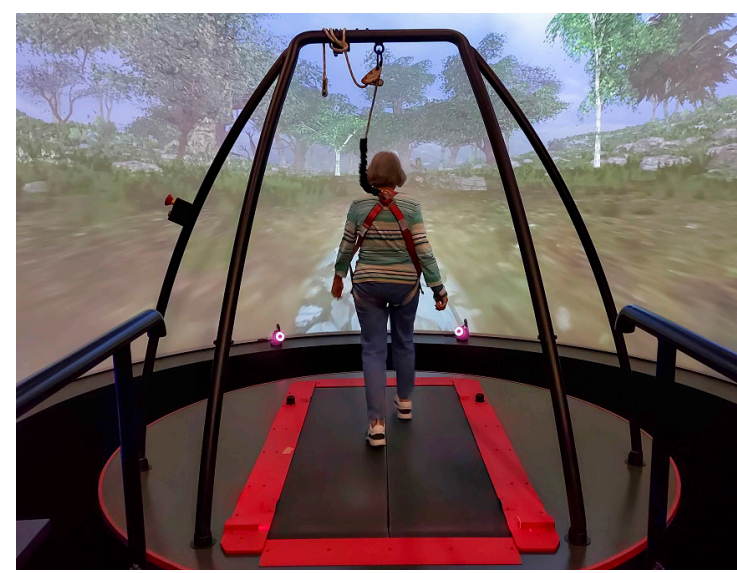

Figure 1 Picture of a participant during perturbation-based balance training. Picture published with the participant's permission.

\section{Interview procedure}

The interviews took place at the hospital, within 1-3 weeks after training completion, between February 2020 and May 2021. Interviews and analysis were completed before results on the effectiveness of the PBT protocol were analysed. The first 13 interviews (of the total 16) were led by a researcher (MHGG) who was involved in the PBT programme because she was familiar with the specifics of the training and could ask targeted follow-up questions. A second researcher was always present at the interviews as an observer to help keep track of the interview guide and take field notes. A verbal summary was given at the end of each interview and a written summary was sent to each participant for a member check. Interviews were conducted iteratively; the interview guide was adjusted after the first three interviews to include emerging themes. This process was repeated after 10 and 13 interviews were completed. All interviews were recorded using a digital voice recorder and transcribed verbatim. Any names and other possible identification information were removed from the transcripts. As the interviews and transcripts were in Dutch, all quotes presented were translated with care to conserve the original meaning.

\section{Analysis}

Interview transcripts were analysed using a template analysis approach. ${ }^{1718}$ The a priori template was formed based on the TFA and interview guide. Two researchers (MHGG and JS) independently coded the first three interviews using NVivo V.12. ${ }^{19}$ After coding, they discussed the codes until consensus and formed an initial template. This process was repeated for the next three interviews. Consensus was then reached that this third template version covered the transcripts that were analysed so far. MHGG subsequently coded the remaining interviews using the third template version. MHGG and JS discussed if any changes needed to be made to the template based on the later interviews. After the ninth interview was coded, no more changes to the template were indicated. This was confirmed by coding the remaining four interviews, and coding saturation was reached. ${ }^{20}$

\section{Reflexivity and triangulation}

As MHGG was involved in the PBT for these participants, as well as sampling and interviewing, it was possible that there was researcher bias or that participants were more inclined to give desirable answers. For the purpose of interviewer triangulation, three additional interviews (interviews 14-16) were conducted by an independent researcher (AW) to confirm or reject previous responses. Coding of these three interviews revealed no new codes, which can be interpreted as secondary confirmation of

Table 1 Theoretical framework of acceptability themes and interpretation

\begin{tabular}{ll}
\hline Theme & Interpretation \\
\hline Affective attitude & How an individual feels about the PBT. \\
Self-efficacy & The participant's confidence in their ability to perform the PBT. \\
& How did you do in the training? \\
\hline Perceived effectiveness & $\begin{array}{l}\text { The extent to which the participant perceives the PBT to potentially and actually (observed) be } \\
\text { effective. }\end{array}$ \\
& To what extent did you experience effects from the training? \\
\hline Ethicality & $\begin{array}{l}\text { The extent to which the intervention had good fit with an individual's value system and } \\
\text { expectations of a falls prevention intervention. }\end{array}$ \\
& To what extent did the training fit with your views on falls prevention? \\
Intervention coherence & $\begin{array}{l}\text { The extent to which the participant understands the aim of PBT and how it works. } \\
\text { In your own words, what was the aim of the training? }\end{array}$ \\
\hline Burden & $\begin{array}{l}\text { The perceived amount of effort that was required to participate in the PBT. } \\
\text { To what extent did you find the training strenuous? To what extent did you find the training } \\
\text { challenging? }\end{array}$ \\
\hline Opportunity costs & The extent to which benefits, profits or values were given up to participate in the PBT. \\
& To what extent did you forego other opportunities to participate in the training?
\end{tabular}

PBT, perturbation-based balance training. 
Table 2 Overview of participant and interview characteristics

\begin{tabular}{llllll}
\hline Participant & Sex & Age (years) & $\begin{array}{l}\text { Falls in the previous } \\
\text { year }(\mathbf{n})\end{array}$ & Interview type & Interviewer \\
\hline P022 & F & 79 & 1 & Face-to-face & MHGG \\
P023 & F & 80 & 1 & Face-to-face & MHGG \\
\hline P026 & M & 65 & 1 & Telephone & MHGG \\
P027 & F & 76 & 2 & Face-to-face & MHGG \\
\hline P030 & F & 83 & 1 & Telephone & MHGG \\
\hline P031 & M & 67 & 1 & Telephone & MHGG \\
P032 & F & 79 & 1 & Telephone & MHGG \\
P040 & F & 73 & 2 & Face-to-face & MHGG \\
P042 & F & 65 & 2 & Face-to-face & MHGG \\
P043 & F & 74 & 1 & Face-to-face & MHGG \\
P044 & F & 79 & 4 & Face-to-face & MHGG \\
P045 & F & 74 & 1 & Face-to-face & MHGG \\
P050 & F & 79 & 1 & Face-to-face & MHGG \\
P068 & F & 70 & 1 & Telephone & AW \\
\hline P069 & F & 66 & 3 & Telephone & AW \\
\hline P082 & F & 69 & 1 & Telephone & AW \\
\hline
\end{tabular}

F, female; M, male.

the coding template and saturation. Participants did not give different answers to different interviewers. JS and AW were not involved in the RCT on PBT and represented an outsider perspective in the design and review of the interview guide, coding and analysis, therewith contributing to further researcher triangulation. Through comparison and discussion of the transcripts, the authors then reached consensus on the most important themes from the interviews.

\section{Patient and public involvement}

Patients were first involved in pilot testing of the PBT protocol in this research and the training protocol was fine-tuned based on their feedback. The aim of this study is to explore participants' perceptions and views on the acceptability of the training, which may be used to guide design or implementation of future PBT interventions.

\section{RESULTS}

\section{Participant description}

Sixteen participants (14 women and 2 men, mean age $73.6 \pm 6.0$ years) were approached, all of whom accepted. For comparison, the RCT included a total of 82 participants, of whom 39 were randomised to the PBT group (median age 73 years (IQR 10 years), 31 women and 8 men). Table 2 provides an overview of the participants and characteristics. Nine interviews were conducted at the hospital and seven were telephone interviews due to restrictions related to the COVID-19 pandemic. The interviews lasted 15-35 min. One participant (P031) missed one training session due to COVID-19 restrictions.
Training adherence was $93.7 \%$ for the full PBT group and 98.3\% for participants included in this qualitative study.

\section{Perceptions of acceptability}

The findings are presented for each of the TFA components, along with illustrative quotes from participants. The theme 'training setting' was added; this theme includes context-specific barriers and facilitators that were described by the participants.

\section{Affective attitude}

Overall, participants described that they felt the PBT was an enjoyable experience. Most participants positively related this to the novelty of the experience, reporting feelings of curiosity and excitement. In contrast, some participants related this novelty to a feeling of suspense and sometimes feeling unsure about their ability to participate in PBT at the start of the first training session. Most often this was described as a good amount of suspense and curiosity about what would happen, and not perceived as a barrier.

I didn't know what I could expect. But I said: Guys, I'll just see what happens, I'll leave it up to you. (...) In the beginning, you don't know what is going to happen to you. You feel a little insecure. But I was glad that I did it. (P044)

One participant described feeling anxious during the first training session, a feeling that fortunately decreased throughout the following sessions.

Now you know what you have to do so it's different. But at first it's kind of a startle response I think. (P030) 
All participants reported that feeling safe and able to challenge themselves without fear of falling was closely related to a positive experience. The safety equipment (especially the safety harness), feeling heard by the trainer and receiving information during the training session were identified as important facilitators.

And again, (laughing) I was very happy that I didn't need to hang from those ropes [safety harness], but I had complete faith that if anything were to happen I definitely would not fall. So that didn't cause any anxiety for me. (P050)

\section{Self-efficacy}

All participants described that they were able to participate in the training sessions and that they felt they did well. Some participants reported that their sense of selfefficacy grew throughout the training sessions, starting with feeling unsure about their ability at the first training session to feeling accomplished after the second or third session. Some of these participants related their initial apprehension to comorbidities.

I have COPD and you're aware that there are situations in which you may have to drop out. And in that sense, this could have happened to me in this training as well. Fortunately, it didn't. (P031)

Finally, one participant provided an example of how she felt after the training sessions.

Well, I can say that I feel I did well. It brings you joy if you have something like that (decreased strength in one leg due to a comorbidity) and you're still able to catch yourself well. (P022)

\section{Perceived effectiveness}

This construct is understood as the extent to which participants perceived changes in their physical or psychological functioning and attributed this to the training. Perceived training effects can be divided into physical and psychological effects. For most participants, recognising physical training effects was not straightforward. Some participants clearly described improvements in their daily activities which they attributed to the training, such as improved walking ability or balance.

I've been walking my son's dog a lot lately. (...) I've noticed that because of that (training) I'm steadier on my legs. Like this morning when I walked him, I had to walk downhill. And before I did the balance training, I would have thought 'Oh I have to be careful', but now I just know: I have to move like this, I have to put my foot here. And I can do this. (P068)

Still, most participants expressed that their physical abilities had remained the same and wondered how they could have noticed possible changes due to training. For most, falling or stumbling was not a daily occurrence to begin with, and as the interviews took place shortly after training completion, they noted that potential changes were not easily identifiable in this short term. This was neither described as a barrier nor a facilitator to participating in the PBT.

But if my balance has improved because of it, that's very hard to determine, because I don't fall very often. That I broke my wrist due to a fall was more of an accident. (P031)

Psychological effects were described as much more apparent and positively related to the acceptability of the intervention. Most participants expressed that the training had helped them gain confidence and improved self-efficacy during the training sessions and in everyday life. They attributed this to how during the training they experienced that their body was capable of more than they expected.

Especially the first time I noticed that I was quite insecure. (...) And that was more related to my confidence, which had been damaged. And I noticed after a few times that I, because of the training actually, gained some confidence. That I got more confident in my body. (P068)

\section{Ethicality}

This construct may not only be related to the extent to which the PBT was perceived to be a good fit with the participant's value system, but also to their expectations of the PBT. Most participants reported that anything they could do to prevent future fall incidents was viewed as valuable.

Well, I was really glad about it, because I thought 'anything I can practice or do to help me fall less, will be helpful'. (P069)

Some participants found it hard to describe if falls prevention in general fit within their value system. Often participants related this to not having heard or thought about falls prevention until they were approached by the study team.

I hadn't heard or read anything about it before, I started this without expectations. (P032)

Some also described having thought about it but not knowing who to approach about the topic or not considering that they needed it. The lack of prior knowledge or expectations about the PBT was not perceived as a barrier to participating. Conclusively, some participants valued being able to contribute to a scientific study.

\section{Intervention coherence}

All participants were able to recognise and describe the aim of the intervention to a certain degree. For example:

In my own words? That you're more able to keep your balance. And it has worked. (P023) 
Another participant provided a more detailed explanation of what she perceived as the aim of the intervention.

To be able to recover, when you've lost balance. I think that that was the aim. That you're able to react; your body, your legs, or even with the help of an arm swing. Faster recovery to regain your balance. (P043)

A factor that repeatedly emerged related to coherence of the intervention is 'intervention validity', understood as the extent to which participants perceived that the intervention had a good fit with its aims. Most participants who discussed this topic described the intervention as valid and perceived this as a facilitator to participating in PBT.

A few times I nearly fell, but then you're able to correct this and it's a beautiful simulation of what can happen in real life. Especially when the treadmill belts don't run at the same speed, when one decelerates while the other continues. Then you get an effect like you're experiencing a slip. (P026)

However, a few participants also questioned if it was at all possible to prevent a future fall incident, reasoning that a fall occurs too sudden to make any preventive adjustments.

\section{Burden}

Participants agreed that the burden of participating in PBT was acceptable. The training was perceived as challenging, but not too challenging. Some participants positively related this to the way the training sessions were structured, providing a gradual and personalised increase in challenge.

No, there was a good and gradual increase in challenge. They started the training quite easy and then it gradually became harder. It was very well structured. (P069)

Most effort was required to maintain or regain balance and to stay focused throughout the session. Participants reported that the required physical stamina was not an important contributing factor to the perceived training load. When mentioned, participants described that the unexpected balance perturbations were perceived as more challenging than the first part of the training, where they could anticipate on what would happen next.

The hard part was when it was sudden, unexpected. Going left, right, forward. That was, well, not hard; you can get it done, but you have to make sure you don't fall, even if you're in a safety harness. (P022)

\section{Opportunity costs}

Participants agreed that no activities had to be given up to participate in the PBT. Most related this to having enough time after retirement. The possibility to schedule the training sessions in consultation with the trainer instead of having fixed training times was reported as an important facilitator. Most participants accepted travelling to the training location as a fact and did not describe this as either a barrier or a facilitator. A few participants found the central location of the hospital a positive factor as this was easy to reach using public transportation. Another few participants described that any location outside walking distance provided a challenge and a potential barrier as they were no longer able to drive a car and had to rely on public transportation or family members to get there. As for the training location being inside a university hospital, this was mostly perceived as an advantage. Participants often described that they thought this was the logical place where they expected to find the right equipment and expertise for this kind of training. Some also mentioned that they regarded the university hospital as a familiar institute and therefore easy to find.

\section{Training setting}

This additional theme was identified throughout the interviews and includes specific factors related to the setting of the PBT. The PBT took place in a specific setting with specialised technological equipment (see figure 1) inside a university hospital. Most participants described that they experienced the technological training equipment as positive, as a surprising and interesting novelty. One participant explained that she felt slightly overwhelmed when she first saw the training equipment, but this improved when she got a more detailed explanation of what was going to happen and when she experienced the training for herself.

The setting was very surprising. The fact that you're walking on a treadmill in an environment that moves with you. I thought it was a very extraordinary experience. (P031)

Most participants described the virtual environments that were used during training as surprising and positive. A few participants expressed a preference for the first virtual environment (the forest road), describing that this felt more friendly and stimulating than the second, more industrial environment.

The individual nature of this training was clearly valued by some participants, while others were ambivalent about this. Participants who indicated a preference for training individually described that they enjoyed being able to really focus on the training itself without distractions from other people or the environment and to train at their own level. Some also reasoned that this increased the potential of the training to be effective.

This, the balance training, is not something you can do in a group. And I just thought it was very pleasant, because you're focusing on yourself. You're focused on what is going to happen, and you can feel everything that happens (...). And I just think that it is much more pleasant this way, and it will be more helpful. (P068) 
While most participants preferred training individually, some described that they were curious to see how other people performed during the training sessions. Additionally, two participants would have appreciated the opportunity to compare and discuss fall-related experiences with peers.

A Numeric Rating Scale (NRS) was used as a tool to individualise training progression. During the training sessions, participants were regularly asked to score how challenged they felt at that moment, ranging from 0 (not challenged at all) to 10 (highest perceivable challenge). Participants described mixed experiences with this system. Some found the scoring easy and even helpful and described that it helped them gain insight into how they felt at that moment. Others described that they had trouble translating their subjective experience to a number that meant little to them. Overall, the NRS scoring was not perceived as an important barrier or facilitator.

\section{DISCUSSION}

The aim of this study was to evaluate the acceptability of a PBT programme as perceived by older adults using the TFA. Gaining understanding of the acceptability of PBT in older adults with a recent history of falls is imperative to enable and optimise future implementation of PBT in clinical practice.

The results indicate that this PBT protocol is perceived as acceptable by older adults with a recent history of falls and highlight key areas for potential future modifications. Besides the TFA, one additional theme emerged from the data, which includes challenges specific to the training setting, such as preference for group training in some participants and travel to the training location.

Participants valued being able to feel safe during training. Most reported that this was accomplished by using the safety harness, the physical presence and guidance from the trainer, and individualised training progression. These results reflect those of Miller $e t a l^{21}$ who evaluated the perceived acceptability of conceptually challenging exercise training to older adults, including dynamic balance tasks with external perturbations. The novelty of the training and technology was regarded as a positive factor contributing to enjoyment by most participants. However, as in Miller et al $\mathrm{s}^{21}$ study, some participants reported feeling initially apprehensive or anxious towards the new training. Okubo et $a l^{11}$ related elevated anxiety levels during PBT to the unpredictability of the perturbations. We previously hypothesised that a more gradual training progression over multiple sessions may help participants build confidence and alleviate anxiety while still being effective. ${ }^{22}$ This hypothesis is partially confirmed by our current findings, which indicated that self-efficacy improved over time. Participants who initially experienced anxiety also reported that this improved over time, and individualised training progression was reported as a facilitator to feeling safe during training.
As unexpected perturbations are key for task-specific PBT, the trade-off between measures to alleviate anxiety while still achieving the desired training stimulus should be considered in future implementation. Our findings suggest that this may be particularly important at the start of training.

Perceived psychological effects in the form of increased confidence in balance abilities and increased self-efficacy in daily life were often reported. Consistent with previous studies on falls prevention interventions, participants described that the PBT helped them gain insight into their ability level and were pleasantly surprised by their ability. ${ }^{21}{ }^{23}$ This is important as maintaining balance confidence can help avoid undue activity avoidance and subsequent disability. ${ }^{24}$ Moreover, decreased balance confidence has been identified as a predictor of future falls. ${ }^{25}$ While participants generally felt they did well during training, perceived physical effects in daily life were less apparent. Participants questioned how they would notice physical training effects, as falling was not a daily occurrence for them to begin with. In part, this may have been related to the interviews taking place shortly after training completion, leaving little time for participants to experience training effects. However, we hypothesise that this may also be related to the perceived intervention validity and ethicality.

While the topic of perceived intervention validity emerged in most of the interviews, participants' views were mixed. Some described that they perceived the intervention valid as it clearly simulated daily-life balance perturbations. In contrast, other participants did not discuss PBT specifically, rather questioned if it was at all possible to prevent a fall in daily life, describing that falls occurred too sudden to intervene or were 'just accidents that could happen to anybody'. The belief that falls are just bad luck and disbelief that they are preventable are well known from the literature. ${ }^{26-29}$ A review by McInnes and Askie $^{30}$ recommended that these beliefs should be countered prior to intervention. While all participants in our study had recently fallen and agreed to participate in the current intervention, none of them actively sought to participate in falls prevention before. Participants described that they had previously given little or no thought to falls prevention or that they did not think they needed it. This is in line with previous studies indicating that those who have previously fallen are not necessarily more likely to be receptive to falls prevention interventions. ${ }^{31} 32$ Another study described that older adults may reject the idea that they need falls prevention because they regard themselves as healthy and able to manage ${ }^{33}$ PBT being a relatively new intervention, it may be assumed that this intervention is even less known to potential participants, thus increasing the challenge to reach the target population. Some participants reported that being informed by or talking to a health practitioner about the PBT, and being approached by the researchers, prompted them to consider falls prevention or to finally participate. This corroborates findings from Yardley et 
$a l,{ }^{34}$ reporting that a personal invitation by a health practitioner may be a facilitating factor to participation in falls prevention. Our findings suggest that these factors may be particularly important to consider for PBT or any relatively unknown intervention to effectively reach older adults.

Some participants clearly expressed a preference for training individually. Others described that they would have been curious to see how others performed during the training sessions or to share fall-related experiences with others. Promoting the social value of falls prevention interventions has been previously identified as a facilitator. ${ }^{30}$ Additionally, watching or partnering with a peer completing the same exercises may facilitate participation and improve older adults' confidence for their own attempt. ${ }^{21} 3536$ As PBT is currently not suited for a group intervention, it may be considered to provide a medium between individual and group training, while simultaneously addressing the initial apprehension or anxiety experienced by some participants. Specifically, this could be achieved by providing new participants with a video of a peer completing the exercises before their own participation, or combining training sessions of two participants where they can see each other perform and share experiences. Conclusively, hearing experiences from peers who completed the PBT might also improve perceived intervention validity and ethicality for future participants.

Finally, a practical factor that should be considered is travelling to the training location. As is the case with most set-ups for PBT, the equipment used in this programme is not yet available in many locations. Some participants described that while they were able to attend the PBT sessions, travel was a potential barrier. This barrier is well known in this population, as some older adults are no longer able to drive a car themselves and thus depend on family members or public transportation. ${ }^{3437}$

\section{Strengths and limitations}

To our knowledge, this is the first study to use the TFA to examine older adults' perceived acceptability of PBT. Using the TFA enabled a systematic approach to define and assess intervention acceptability. ${ }^{9}$ While triangulation was applied in data collection as well as data analysis to increase trustworthiness of the research findings, it should be noted that one researcher (MHGG) was involved in the PBT sessions, as well as most of the interviews. In future studies, it may be considered to include a dedicated interviewer separate to the intervention team. A few limitations should be considered when interpreting the results of our study. First, the PBT intervention was applied in a research setting, meaning that some specific factors, such as willingness to pay for participation in the intervention, were not evaluated. Second, the results only reflect the perceived retrospective acceptability of the PBT. Further research is necessary to evaluate prospective and concurrent acceptability to further elucidate what factors motivate or prevent older adults from participating in PBT. $^{9}$
A final consideration is that due to COVID-19-related restrictions, 7 out of the 16 interviews in this study were telephone interviews. While face-to-face interviews are often regarded as the gold standard, there is little evidence that quality of findings collected through telephone interviews is compromised.$^{38}$ Consistent with Sturges and Hanrahan, ${ }^{39}$ we identified no clear differences between data collected through face-to-face interviews and with telephone interviews in our study.

\section{CONCLUSIONS}

In conclusion, findings from this study indicate that a technology-assisted PBT programme is acceptable to older adults with a recent falls history. Enjoyment of the intervention, being able to feel safe, perceived psychological effectiveness and individualised training progression were identified as important factors contributing to the perceived acceptability. Increasing the social aspect of training and sharing experiences of peers may be considered to enhance acceptability to new participants who initially feel apprehensive or anxious about their ability to participate or who are unsure what to expect. Raising awareness of the importance and possibilities of falls prevention training in general is a challenge in this population.

\section{Author affiliations}

${ }^{1}$ Department of Physical Therapy, Maastricht University Medical Center+, Maastricht, The Netherlands

${ }^{2}$ Department of Epidemiology, Care and Public Health Institute (CAPHRI), Maastricht University, Maastricht, The Netherlands

${ }^{3}$ Department of Anatomy and Embryology, Maastricht University, Maastricht, The Netherlands

${ }^{4}$ Department of Nutrition and Movement Sciences, School of Nutrition and Translational Research in Metabolism (NUTRIM), Maastricht University, Maastricht, The Netherlands

Acknowledgements We thank Anouk Weemaes for her contribution to conducting the interviews in this study.

Contributors MHGG: conceptualisation, methodology, validation, formal analysis, investigation, data curation, writing - original draft, visualisation, project administration, guarantor. JS: methodology, validation, formal analysis, data curation, writing - review and editing. RM: conceptualisation, writing - review and editing. RAdB, KM: conceptualisation, writing - review and editing, supervision. AFL: conceptualisation, writing - review and editing, supervision, project administration.

Funding The authors have not declared a specific grant for this research from any funding agency in the public, commercial or not-for-profit sectors.

Competing interests None declared.

Patient consent for publication Obtained.

Ethics approval This study involves human participants and was approved by the Medical Ethics Committee of MUMC+ (Maastricht University Medical Center) and Maastricht University (METC NL67131.068.18). Participants gave informed consent to participate in the study before taking part.

Provenance and peer review Not commissioned; externally peer reviewed.

Data availability statement Data are available upon reasonable request.

Supplemental material This content has been supplied by the author(s). It has not been vetted by BMJ Publishing Group Limited (BMJ) and may not have been peer-reviewed. Any opinions or recommendations discussed are solely those of the author(s) and are not endorsed by BMJ. BMJ disclaims all liability and responsibility arising from any reliance placed on the content. Where the content includes any translated material, BMJ does not warrant the accuracy and reliability 
of the translations (including but not limited to local regulations, clinical guidelines, terminology, drug names and drug dosages), and is not responsible for any error and/or omissions arising from translation and adaptation or otherwise.

Open access This is an open access article distributed in accordance with the Creative Commons Attribution Non Commercial (CC BY-NC 4.0) license, which permits others to distribute, remix, adapt, build upon this work non-commercially, and license their derivative works on different terms, provided the original work is properly cited, appropriate credit is given, any changes made indicated, and the use is non-commercial. See: http://creativecommons.org/licenses/by-nc/4.0/.

\section{ORCID IDs}

Marissa H G Gerards http://orcid.org/0000-0001-7388-2356

Antoine F Lenssen http://orcid.org/0000-0003-3627-4452

\section{REFERENCES}

1 Sherrington C, Fairhall NJ, Wallbank GK, et al. Exercise for preventing falls in older people living in the community. Cochrane Database Syst Rev 2019;1:CD012424.

2 Rieger MM, Papegaaij S, Steenbrink F, et al. Perturbation-Based gait training to improve daily life gait stability in older adults at risk of falling: protocol for the react randomized controlled trial. BMC Geriatr 2020;20:167.

3 Bunn F, Dickinson A, Barnett-Page E, et al. A systematic review of older people's perceptions of facilitators and barriers to participation in falls-prevention interventions. Ageing Soc 2008;28:449-72.

4 Berg WP, Alessio HM, Mills EM, et al. Circumstances and consequences of falls in independent community-dwelling older adults. Age Ageing 1997;26:261-8.

5 Talbot LA, Musiol RJ, Witham EK, et al. Falls in young, middleaged and older community dwelling adults: perceived cause, environmental factors and injury. BMC Public Health 2005;5:86.

6 Maki BE, Mcllroy WE. Change-in-support balance reactions in older persons: an emerging research area of clinical importance. Neurol Clin 2005;23:751-83.

7 Mansfield A, Wong JS, Bryce J, et al. Does perturbation-based balance training prevent falls? Systematic review and meta-analysis of preliminary randomized controlled trials. Phys Ther 2015;95:700-9.

8 Pai Y-C, Bhatt T, Yang F, et al. Perturbation training can reduce community-dwelling older adults' annual fall risk: a randomized controlled trial. J Gerontol A Biol Sci Med Sci 2014;69:1586-94.

9 Sekhon M, Cartwright M, Francis JJ. Acceptability of healthcare interventions: an overview of reviews and development of a theoretical framework. BMC Health Serv Res 2017;17:88.

10 Osho O, Owoeye O, Armijo-Olivo S. Adherence and attrition in fall prevention exercise programs for community-dwelling older adults: a systematic review and meta-analysis. J Aging Phys Act 2018;26:304-26.

11 Okubo Y, Brodie MA, Sturnieks DL, et al. A pilot study of reactive balance training using trips and slips with increasing unpredictability in young and older adults: biomechanical mechanisms, falls and clinical feasibility. Clin Biomech 2019;67:171-9.

12 Kurz I, Gimmon Y, Shapiro A, et al. Unexpected perturbations training improves balance control and voluntary stepping times in older adults - a double blind randomized control trial. BMC Geriatr 2016;16:58

13 Lurie JD, Zagaria AB, Pidgeon DM, et al. Pilot comparative effectiveness study of surface perturbation treadmill training to prevent falls in older adults. BMC Geriatr 2013;13:49.

14 Mansfield A, Peters AL, Liu BA, et al. Effect of a perturbation-based balance training program on compensatory stepping and grasping reactions in older adults: a randomized controlled trial. Phys Ther 2010;90:476-91.

15 Tong A, Sainsbury P, Craig J. Consolidated criteria for reporting qualitative research (COREQ): a 32-item checklist for interviews and focus groups. Int J Qual Health Care 2007;19:349-57.

16 Gerards MHG, Marcellis RGJ, Poeze M, et al. Perturbation-based balance training to improve balance control and reduce falls in older adults - study protocol for a randomized controlled trial. BMC Geriatr 2021;21:9.

17 Brooks J, McCluskey S, Turley E, et al. The utility of template analysis in qualitative psychology research. Qual Res Psychol 2015;12:202-22

18 King N, analysis T. Qualitative methods and analysis in organizational research: a practical guide. Thousand Oaks, CA: Sage Publications Ltd, 1998: 118-34.

19 QSR International Pty Ltd. NVivo qualitative data analysis software, version 12, 2018

20 Saunders B, Sim J, Kingstone T, et al. Saturation in qualitative research: exploring its conceptualization and operationalization. Qual Quant 2018:52:1893-907.

21 Miller CT, Teychenne M, Maple J-L. The perceived feasibility and acceptability of a conceptually challenging exercise training program in older adults. Clin Interv Aging 2018;13:451-61.

22 Gerards MHG, McCrum C, Mansfield A, et al. Perturbation-Based balance training for falls reduction among older adults: current evidence and implications for clinical practice. Geriatr Gerontol Int 2017;17:2294-303

23 Davis JC, Dian L, Parmar N, et al. Geriatrician-led evidencebased falls prevention clinic: a prospective 12-month feasibility and acceptability cohort study among older adults. BMJ Open 2018;8:e020576.

24 Büla CJ, Monod S, Hoskovec C, et al. Interventions aiming at balance confidence improvement in older adults: an updated review. Gerontology 2011;57:276-86.

25 Landers MR, Oscar S, Sasaoka J, et al. Balance confidence and fear of falling avoidance behavior are most predictive of falling in older adults: prospective analysis. Phys Ther 2016;96:433-42.

26 Commonwealth of Australia. Preventing falls and harm from falls in older people: best practice guidelines for Australian community care, 2009. Available: https://www.safetyandquality.gov.au/sites/default/ files/migrated/Guidelines-COMM.pdf [Accessed 19 Aug 2021].

27 Ballinger C, Payne S, Claire Ballinger SP. Falling from grace or into expert hands? Alternative accounts about falling in older people. $\mathrm{Br} \mathrm{J}$ Occup Ther 2000;63:573-9.

28 Kong KS-wai, Lee Fk F-kam, Mackenzie AE, et al. Psychosocial consequences of falling: the perspective of older Hong Kong Chinese who had experienced recent falls. J Adv Nurs 2002;37:234-42.

29 Finnegan S, Bruce J, Seers K. Life after falls prevention exercise - experiences of older people taking part in a clinical trial: a phenomenological study. BMC Geriatr 2021;21:91.

30 Mclnnes E, Askie L. Evidence review on older people's views and experiences of falls prevention strategies. Worldviews Evid Based Nurs 2004;1:20-37.

31 Cumming RG, Thomas M, Szonyi G, et al. Adherence to occupational therapist recommendations for home modifications for falls prevention. Am J Occup Ther 2001;55:641-8.

32 Edwards N, Lockett D, Aminzadeh F, et al. Predictors of Bath Grab-Bar use among Community-Living older adults. Can J Aging 2003;22:217-27.

33 Allen A, Simpson JM. A primary care based fall prevention programme. Physiother Theory Pract 1999;15:121-33.

34 Yardley L, Donovan-Hall M, Francis K, et al. Attitudes and Beliefs That Predict Older People's Intention to Undertake Strength and Balance Training. J Gerontol B Psychol Sci Soc Sci 2007;62:P119-25.

35 Bethancourt HJ, Rosenberg DE, Beatty T, et al. Barriers to and facilitators of physical activity program use among older adults. Clin Med Res 2014:12:10-20.

36 Biedenweg K, Meischke H, Bohl A, et al. Understanding older adults' motivators and barriers to participating in organized programs supporting exercise behaviors. J Prim Prev 2014;35:1-11.

37 Kiami SR, Sky R, Goodgold S. Facilitators and barriers to enrolling in falls prevention programming among community dwelling older adults. Arch Gerontol Geriatr 2019;82:106-13.

38 Novick G. Is there a bias against telephone interviews in qualitative research? Res Nurs Health 2008;31:391-8.

39 Sturges JE, Hanrahan KJ. Comparing telephone and face-to-face qualitative interviewing: a research note. Qualitative Research 2004;4:107-18. 